\title{
Intransparenz, Mitbrüderlichkeit, mangelnde Konsequenz
}

\author{
Umgang mit einem pädophilen Priester im Bistum Münster (1958-2007)
}

\author{
Bernhard Frings
}

Der ,Fall Pottbäcker ' gilt im Bistum Münster als „Menetekel“", also als ernster Mahnruf. Ausgelöst durch eine Gemeindeveranstaltung im November 2018 im münsterländischen Rhede, auf der die Anfang der 1970er Jahre in der Gemeinde verübten Missbrauchstaten des damaligen Kaplans erstmals öffentlich diskutiert wurden, kam nachfolgend eine breite Berichterstattung auch in den überregionalen Medien in Gang. ${ }^{2}$ Fortan stand Pottbäcker „für den schändlichen sexuellen Missbrauch schutzbefohlener Kinder durch einen pädophilen Kaplan und das Versagen der Amtskirche, die über Jahre den Täter schützte und die Opfer ignorierte“, wie es in einem Zeitungsartikel hieß. ${ }^{3}$ Dabei geriet auch der langjährige, 2013 verstorbene Diözesanbischof Reinhard Lettmann derart in den Fokus, dass die Gremien seiner Heimatpfarrei im Sommer 2019 beschlossen, dem nach ihm benannten Gemeindehaus einen anderen Namen zu geben. ${ }^{4}$

Aber der ,Fall Pottbäcker' ist nicht nur der eingangs zitierte Mahnruf, sondern auch ein - sicherlich prominentes - Beispiel, an dem sich die Strukturen und Mechanismen des Umgangs der Personalverantwortlichen im Bistum Münster mit einem übergriffigen, als pädophil geltenden Priester

1 Vgl. Artikel „Sexueller Missbrauch im Bistum Münster. Kaplan Pottbäckers Taten und das Versagen des Bistums" (Westfälische Nachrichten-Online vom 12.4.2019).

2 Etwa die Beiträge „Pädophiler Priester wird versetzt - und dann rückfällig. Kirche bittet um Vergebung“ (Bild-Zeitung vom 27.11.2018); „Verurteilter pädophiler Priester versetzt: neuer Missbrauch“ (RTL vom 27.11.2018); „Pädophiler Priester versetzt - neuer Missbrauch“ (Spiegel Online vom 27.11.2018); „Bistum: Fehler bei Bischof Lettmann im Umgang mit Missbrauch" (Katholische Nachrichten-Agentur vom 28.11.2018); ,Verurteilter pädophiler Priester 1971 versetzt - weiterer Missbrauch“ (Deutsche Presse-Agentur vom 28.11.2018); „Bistum hielt an pädophilem Priester fest. Zahlreiche Kinder missbraucht“ (n-tv vom 28.11.2018); „Bistum: Bischof Lettmann machte Fehler im Umgang mit Missbrauch“ (katholisch.de vom 28.11.2018); „Bistum hat Täter lebenslang gedeckt. Sexuelle Gewalt an Kindern durch Kaplan Heinz Pottbäcker" (Bocholter-Borkener Volksblatt vom 29.11.2018).

3 Artikel „Sexueller Missbrauch im Bistum Münster. Kaplan Pottbäckers Taten und das Versagen des Bistums" (Westfälische Nachrichten-Online vom 12.4.2019).

4 Chronologie der Diskussion auf der Homepage der Katholischen Kirchengemeinde St. Amandus Datteln, einzusehen unter https://www.st-amandus-datteln.de/andenken-anreinhard-lettmann.html, zuletzt aufgerufen am 24.2.2021.

(C) BERNHARD FRINGS, 2022 | DOI:10.30965/9783657791217_014

This is an open access chapter distributed under the terms of the CC BY-NC-ND 4B license. Frings - 9783657791217 
nachzeichnen lassen. Konkret geht es um spezifische Wege der Vertuschung und Desinformation, um die Unfähigkeit, Missbrauchshandlungen wirklich konkret zu benennen, um das große Vertrauen in eine therapeutische Behandlung, um Kontrollstrategien, um Mitbrüderlichkeit als ein wesentliches handlungsleitendes Moment und schließlich um eine Art Kapitulation vor der - in heutiger Terminologie - pädosexuellen Präferenzstörung, der der Bischof zunehmend mit Hilflosigkeit begegnete. Quasi überwölbt werden alle diese Aspekte jedoch durch den aus heutiger Sicht völlig unzureichenden Blick auf die vom Missbrauch Pottbäckers betroffenen Kinder, deren Schicksal - so scheint es zumindest - bei allen Reaktionen und eingeleiteten Schritten nur am Rande eine Rolle spielte.

Vor allem auf Basis der vergleichsweise dicht geführten Personalakte und der Missbrauchsakte Pottbäckers, aber auch zahlreicher Zeitungsartikel und einiger anderer Veröffentlichungen sowie von Interviews und Hintergrundgesprächen mit bislang drei Betroffenen, zwei Zeitzeugen sowie einer Pottbäcker nahestehenden Person sollen nachfolgend zunächst die Missbrauchstaten - wo möglich auch aus der Betroffenenperspektive - beschrieben werden, um dann ausführlich auf das jeweilige Agieren der Bistumsleitung einzugehen. Dabei handelt es sich noch um vorläufige Ergebnisse der an der Universität Münster angesiedelten Studie zur Aufarbeitung des sexuellen Missbrauchs im Bistum Münster, die bis zum Frühjahr 2022 angelegt ist. ${ }^{5}$

\section{Jahrzehnte des sexuellen Missbrauchs (1967-1983)}

1937 am Niederrhein geboren und nach dem Theologiestudium in Münster und Innsbruck 1964 zum Priester geweiht, durchlief Heinz Pottbäcker während seines 43-jährigen Priesterlebens 14 Stationen. Bei vielen Versetzungen spielten seine pädosexuellen Präferenzstörungen eine maßgebliche Rolle, ohne dass dabei stets konkrete Vergehen bzw. Verbrechen ${ }^{6}$ bekannt geworden sind. Immerhin ermittelte 1967/68 und 1983 die Staatsanwaltschaft wegen sexuellen

5 Projektinformationen einzusehen unter http://go.wwu.de/missbrauchsstudie, zuletzt aufgerufen am 24.2.2021. Zum Bistum Münster nach 1945 vgl. Werner Thissen (Hrsg.), Das Bistum Münster, 3 Bde. Münster 1993; Willi Baumann (Hrsg.), Die katholische Kirche im Oldenburger Land. Ein Handbuch. Vechta 1995; Wilhelm Damberg, Abschied vom Milieu? Katholizismus im Bistum Münster und in den Niederlanden 1945-198o. Paderborn u.a. 1997.

6 Als Vergehen gelten rechtswidrige Taten, die eine geringe Freiheitsstrafe oder eine Geldstrafe zur Folge haben, wohingegen Verbrechen eine Mindestfreiheitsstrafe (heute ein Jahr) nach sich ziehen. 
Missbrauchs von Minderjährigen gegen Pottbäcker. ${ }^{7}$ Beide Daten grenzen auch den Zeitraum ein, in dem der Geistliche darüber hinaus nachweisbar Übergriffe an zahlreichen weiteren Jungen, aber auch an Mädchen verübte. Dazu boten sich ihm in den jeweiligen Pfarreien durch sein großes Engagement in der Jugendseelsorge und Jugendarbeit vielfältige Möglichkeiten.

So erteilte er 1967 laut den staatsanwaltschaftlichen Ermittlungsergebnissen als Kaplan in Waltrop - wegen Unstimmigkeiten mit dem Pfarrer seiner ersten Kaplanstelle im niederrheinischen Aldekerk war er bereits Anfang 1966 nach nur einem Jahr in das nördliche Ruhrgebiet gewechselt - einem neunjährigen Jungen Einzel-Kommunionunterricht. Außerdem besuchte der Junge gemeinsam mit anderen Kindern Pottbäcker in dessen Wohnung, ,weil sie dort gemeinsam sangen und Gitarre spielten“. Kurz vor Ostern erhielt er - diesmal gemeinsam mit seiner Schwester - erneut eine katechetische Unterweisung, wobei er auf dem Schoß des Kaplans saß. Durch ein Loch in der Hosentasche des Jungen spielte Pottbäcker längere Zeit mit dessen Geschlechtsteil. Auf Grundlage des „glaubhaften Geständnis[ses] des Angeklagten“ verurteilte das Landgericht Recklinghausen Pottbäcker „wegen Unzucht mit einem abhängigen männlichen Kind“ - im Detail wegen Missbrauchs an Schutzbefohlenen ( $(174)$ bzw. Kindern $(\S 176)$ sowie homosexuellen Handlungen (§ 175) - zu einer Gefängnisstrafe von neun Monaten. Da die Richter davon ausgingen, dass Pottbäcker „unter der Einwirkung einer Strafaussetzung in Zukunft ein gesetzmässiges und geordnetes Leben führen" werde, setzten sie die Strafe auf Bewährung aus. ${ }^{8}$

Auch die Personalverantwortlichen in Münster hatten offenbar keine Vorbehalte, Pottbäcker wenig später wieder eine Aufgabe in einer Gemeinde zu geben, sodass er erneut Gelegenheit erhielt, im Rahmen seiner seelsorglichen Tätigkeiten Kinder zu missbrauchen. Als Kaplan in Bockum-Hövel (1968-1971) verübte er schon bald nach seiner Ankunft bei Ferienfreizeiten, der Betreuung des Nachsitzens in einer Schule oder bei Autofahrten in seinem Käfer weitere Taten. Während einer Fahrt in einen Winterurlaub nach Österreich sei es nach der Schilderung Betroffener sogar zu einem Unfall gekommen, wobei vermutlich einer der Jungen auf Pottbäckers Schoß gesessen habe. ${ }^{9}$

7 Vgl. Anklageschrift der Oberstaatsanwaltschaft Bochum vom 15.12.1967 und Urteil des Landgerichts Recklinghausen vom 6.6.1968 (Missbrauchsakte, Bistumsarchiv Münster (BAM), Bischöfliches Generalvikariat (BGV) Münster, Hauptabteilung Seelsorge-Personal (HA 500), Reg. A ooog, Bl. 45-52); Strafbefehl gegen Pottbäcker o. Dat. [Übersendung an das BGV Münster am 28.6.1983] (ebd., Bl. 41ff.).

8 Urteil vom 6.6.1968 (ebd., Bl. 45-48).

9 Vgl. mehrere Betroffenenmeldungen in der Missbrauchsakte von Januar bis Juni 2019 (ebd., Bl. 2f. und BAM, BGV Münster, HA 5oo, Reg. A ooo6, Bl. 37f.) und Artikel „Das System des 
Als Kaplan in Rhede (1971-1973) knüpfte Pottbäcker an seine Anbahnungsstrategie der engagierten Jugendarbeit an, wobei besonders Messdiener und Teilnehmer der Ferienfreizeiten vom Missbrauch betroffen waren. Alleine schon durch sein Gitarrenspiel im Gottesdienst habe er geradezu einen „Hype“ ausgelöst, wie es ein Betroffener formuliert. ${ }^{10}$ Denn obwohl im Zuge der Liturgiereform des Zweiten Vatikanischen Konzils (1962-1965) in vielen Kirchen die Zelebrationsaltäre in die Mitte des Chorraums gerückt waren und der Geistliche die Messtexte anstatt in Latein nun meist in Deutsch sprach, also die tätige Teilnahme der Gläubigen am Gottesdienst ermöglicht werden sollte, dominierte Anfang der 197oer Jahre noch weitgehend die vorkonziliare Priestergeneration, die zudem häufig ihre abgehobene Stellung betonte. ${ }^{11}$ Auch sonst sei Pottbäcker „von seiner Art her anders als alle Erwachsenen“ gewesen. Er habe die Kinder ernst genommen und eine durchaus "charismatische“ Ausstrahlung besessen. ${ }^{2}$

So trafen sich nach den Erinnerungen Betroffener auch Kinder und Jugendliche in Pottbäckers Wohnung, um zu spielen, Schallplatten zu hören oder exotische Instrumente zu spielen. Dabei streichelte er die jungen Besucher und verteilte offen „Küsschen“. Durch diese Annährungen wollte er vermutlich nicht zuletzt testen, „wie weit er gehen kann“.13 Jedenfalls fand Pottbäcker eine Reihe von Kindern - darunter auch wenigstens zwei Mädchen -, die er teils über mehrere Jahre in regelmäßigen Abständen missbrauchte, wobei sein Büro und Unterkünfte in Ferienlagern, aber auch die Sakristei zu den Tatorten zählten. Die pädosexuellen Verbrechen umfassten vor allem Berührungen im Genitalbereich zur eigenen Stimulation, aber auch Masturbation und orale Befriedigung durch die Betroffenen. Einer von ihnen erinnert sich etwa, dass der Missbrauch immer im Büro Pottbäckers stattfand, wenn er dort alle 14 Tage samstags vor der Vorabendmesse seinen Ministranten-Dienstplan abholte. Stets musste er sich dann bei Pottbäcker auf den Schoß setzen, der schon bald seine Hand unter die Hose des Jungen schob und sich dann selbst befriedigte. Deutlich steht dem Betroffenen noch vor Augen, dass zudem immer der im Büro stehende Fernseher lief. Dieses Szenario wiederholte sich ohne spürbare

Vertuschens. Alle haben weggeschaut: Der ,Fall Pottbäcker und die Opfer aus der Bockum-Höveler Christus-König-Gemeinde“ (Westfälischer Anzeiger vom 8.12.2018). Interview Franz Jeschke (Pseudonym) vom 24.8.2020.

11 Vgl. etwa Klemens Richter, Liturgiereform - eine bleibende Aufgabe. 40 Jahre Konzilskonstitution über die heilige Liturgie. Münster 2004.

12 Interview Martin Schmitz vom 8.9.2020.

13 Ebd. 
Unterbrechung bis zum Weggang Pottbäckers, sodass er von ca. 70 erlittenen Missbrauchstaten ausgeht. ${ }^{14}$

Betroffenenmeldungen und staatsanwaltschaftliche Ermittlungen belegen weitere Übergriffe Pottbäckers in Marl (1973/74) sowie als Pfarrer in Recklinghausen (1981-1983). Aber erst im Frühjahr 1983 musste er sich dafür erneut vor der Justiz verantworten, nachdem ihn die Eltern eines Jungen wegen sexuellen Missbrauchs ihres Sohns angezeigt hatten. ${ }^{15}$ Auch diesmal legte er ein umfassendes Geständnis ab. Demnach hatte er seit September 1982 drei Jungen im Alter zwischen neun und elf Jahren mehrfach jeweils über der Hose am Geschlechtsteil berührt. Ferner fasste er einem Jungen an den nackten Penis und veranlasste ihn dazu, dies ebenfalls bei ihm zu machen. Zudem sei es bei einem anderen zu „beischlafähnliche[n] Bewegungen“ gekommen. Trotzdem verzichtete die zuständige Justizbehörde auf ein öffentliches Verfahren ob ihr die Vorstrafe Pottbäckers bekannt war, ist nicht ersichtlich - und stellte ihm nur einen Strafbefehl zu, in dem eine Geldstrafe von insgesamt gut 12500 DM und die Zahlung der Verfahrenskosten festgesetzt wurden. ${ }^{16}$ Das Rechtsmittel des Strafbefehls wird häufig etwa bei Vergehen im Straßenverkehr oder bei Ladendiebstählen zur Entlastung der Gerichte angewandt. ${ }^{17}$

Wie nachfolgend noch näher erläutert wird, suchte Pottbäcker auch bei seinen weiteren, nicht mehr in der unmittelbaren Pfarrseelsorge angesiedelten Priesterstationen Kontakt zu Kindern. Allerdings geben weder die Akten noch Gespräche mit Betroffenen konkrete Anhaltspunkte, dass es dabei nochmals zu sexuellen Übergriffen gekommen ist. Nach Auswertung der Akten und Zeitungsberichte lassen sich für den Zeitraum 1967-1983 konkrete Hinweise auf 21 Betroffene finden. Allerdings ist von einer sehr hohen Dunkelziffer auszugehen, da Pottbäcker im Rahmen seiner Tätigkeiten in den jeweiligen Pfarreien vielfältige Möglichkeiten der Tatanbahnung und -ausführung hatte. Martin Schmitz, der über zahlreiche, über Rhede hinausgehende Betroffenenkontakte verfügt und allein von elf Betroffenen in seiner Heimatgemeinde Rhede weiß,

\footnotetext{
14 Interview Franz Jeschke (Pseudonym) vom 24.8.2020.

15 Artikel „Anzeige gegen Pfarrer“ (Westdeutsche Allgemeine Zeitung vom 4.5.1983).

16 Strafbefehl gegen Pottbäcker o. Dat. [Übersendung der Kopie an das BGV Münster am 28.6.1983] (Missbrauchsakte, BAM, BGV Münster, HA 5oo, Reg. A ooo9, Bl. 4lff.).

17 Vgl. etwa Klaus Jochen Müller, Das Strafbefehlsverfahren (§§ 407ff. StPO). Eine dogmatisch-kriminalpolitische Studie zu dieser Form des schriftlichen Verfahrens unter besonderer Berücksichtigung der geschichtlichen Entwicklung - zugleich ein Beitrag zum STVÄG 1987. Frankfurt a. M. 1993.
} 
aber von deutlich mehr Personen ausgeht, spricht daher in seiner, wie er es nennt, "realistischen Spekulation“ von 5 o bis 100 Betroffenen. ${ }^{18}$

\section{Fehlendes Wissen und Problembewusstsein der Bistumsverantwortlichen (1958-1973)}

Als Pottbäcker 1958 nach dem Abitur an einem staatlichen Gymnasium als Priesteramtskandidat in das Theologen-Konvikt Collegium Borromaeum nach Münster kam, scheinen bei den Personalverantwortlichen keine Vorbehalte an seiner Eignung für den eingeschlagenen Lebensweg bestanden zu haben. Zumindest gaben die Sittenzeugnisse seines letzten Religionslehrers und seines Heimatpfarrers, die üblicherweise dem Aufnahmegesuch eines Kandidaten beim Bischof beigefügt wurden, keinen Anlass, Pottbäcker mit Skepsis zu begegnen. ${ }^{19}$ So stamme er aus einer "treukatholischen und kirchlich-aktiven Familie“ und habe schon länger den „Wunsch“ gehegt, „Priester zu werden“. Jedenfalls seien beim Lehrer nie Zweifel am „Ernst seines sittlichen und religiösen Strebens" aufgekommen, sodass er „die Voraussetzungen für das Priestertum in jeder Hinsicht gegeben" sah. Ferner sei Pottbäcker bei Lehrern wie Mitschülern „wegen seiner Freundlichkeit und seines gefälligen Wesens“ beliebt gewesen. Einzig seine Unbekümmertheit schien dem Lehrer etwas zu ausgeprägt. ${ }^{20}$

Die Personalakte Pottbäckers gibt nachfolgend nur noch wenige knappe Auskünfte über seine Zeit im Collegium Borromaeum und im Priesterseminar, die kaum Rückschlüsse etwa auf seine Persönlichkeit und Lebensweise ermöglichen. ${ }^{21}$ Ein im gleichen Jahr zum Priester geweihter Mitstudent erinnert sich an keine Gerüchte über Pottbäckers pädosexuelle Präferenzstörungen ${ }^{22}$, und ob diese bereits etwa dem Regens bekannt waren, ist nicht überliefert. Doch wenn der Direktor des Borromaeums davon sprach, dass es der ausdrückliche „Wunsch“ Bischof Michael Kellers gewesen sei, Pottbäcker im Schuljahr 1960/61 als „Assistent“ im bischöflichen Studienseminar Gaesdonck - also als Erzieher im Internatsbereich des Gymnasiums - einzusetzen, ist von keinen

\footnotetext{
18 Interview Martin Schmitz vom 8.9.2020. Auch Martin Schmitz sind für den Zeitraum nach 1983 pädokriminelle Verbrechen Pottbäckers nicht bekannt.

19 Sittenzeugnisse des Heimatpfarrers und Religionslehrers vom 14.2.1958 (Personalakte, BAM, Generalvikariat Neues Archiv (GV NA), HA 500, A 1481, Bl. 139f.)

$20 \quad$ Sittenzeugnis des Religionslehrers vom 14.2.1958 (ebd., Bl. 140).

21 Vgl. Personalakte Pottbäckers.

22 Vgl. Interview Pfarrer B. vom 14.1.2O2O.
} 
Vorbehalten der Personalverantwortlichen gegenüber Pottbäckers „sittlichem“ Verhalten auszugehen. ${ }^{23}$

Allerdings hätten Alarmglocken läuten können, als der Pfarrer der Aldekerker Kirchengemeinde - der ersten Kaplanstelle Pottbäckers - Ende 1965 bei Generalvikar Laurenz Böggering um die Versetzung des jungen Geistlichen bat. Denn in der ausführlichen Auflistung der in den Augen des Pfarrers unzureichenden Arbeitseinstellung und großen Unzuverlässigkeit seines Kaplans - er vernachlässige völlig die Jugendarbeit, und komme permanent zu spät ins Pfarrhaus zurück, weil er in „Lokalen Karten“ spiele - kam auch ein weiterer Kritikpunkt zur Sprache: Pottbäcker gehe in der „Mittagszeit“, wenn Pfarrer und Haushälterin ruhten, aus und hole „Kinder auf sein Zimmer, um ihnen etwas vorzuspielen“. Erst nachdem der Pfarrer "dies strikte verboten“ hatte, habe der Kaplan damit aufgehört. Zwar kündigte Böggering „nach reiflicher Überlegung" schon bald die zeitnahe Versetzung Pottbäckers an, damit „die entstandenen Spannungen hinsichtlich der priesterlichen Zusammenarbeit gelöst" würden. Die große Nähe, die Pottbäcker Kindern gegenüber offenbar auch in privater Umgebung suchte, scheint jedoch nicht besonders aufgefallen zu sein. ${ }^{24}$

Schriftwechsel und pfarrliche Zeugnisse im Zusammenhang mit der turnusgemäßen Wiederholung des „Curaexamen“ (Dienstprüfung) Pottbäckers ${ }^{25}$ verweisen darauf, dass die Bistumsverantwortlichen bis zum Spätsommer/ Herbst 1967 keine Kenntnisse von den pädosexuellen Präferenzstörungen des Kaplans hatten, also seine zuvor vollzogenen Versetzungen von Waltrop zur knapp dreimonatigen Aushilfe nach Bösensell und von dort nach Dinslaken-Lohberg keine Reaktion auf bereits geäußerte Anschuldigungen waren. ${ }^{26}$ Allerdings betonten auch diese Beurteilungen neben der nach wie vor schlechten Arbeitsmoral und den regelmäßigen nächtlichen Ausflügen des Kaplans seine besondere Ausstrahlung auf Kinder und Jugendliche, da er mit ihnen „viel Sport treibt“ und es ihm „als guter Sänger und Musikliebhaber“

23 Bescheinigung des Direktors vom 10.5.196o (Personalakte, Bl. 127). Die Rekrutierung von Priesteramtskandidaten als Internatserziehende in bischöflichen Einrichtungen zählte auch bei den Regensburger Domspatzen zur langjährigen Praxis, vgl. Bernhard Frings/ Bernhard Löffler, Der Chor zuerst. Institutionelle Strukturen und erzieherische Praxis der Regensburger Domspatzen 1945 bis 1995. Regensburg 2019, 21of.

24 Aldekerker Pfarrer an Generalvikar Böggering vom 3.12.1965 und Antwort vom 13.1.1966 (Personalakte, Bl. 115f.).

25 BGV Vorgang 150/67 („Curainstrument“ Pottbäcker) vom 30.5.1967, Bösenseller Pfarrer an BGV vom 13.6.1967 und Zeugnis des Waltroper Pfarrers vom 14.6.1967 (ebd., Bl. 110 und 112f.).

26 BGV an Pottbäcker vom 10.4.1967 und Bösenseller Pfarrer an BGV vom 13.7.1967 (ebd., Bl. 109 und 114). 
schnell gelinge, „Kontakt herzustellen“. ${ }^{27}$ Letztlich wird man davon ausgehen können, dass die Bistumsleitung in Münster erst durch die staatsanwaltschaftlichen Ermittlungen konkrete Hinweise auf Pottbäckers distanzloses Verhalten und sexuelle Übergriffe erhielt.

Es war vor allem der erst seit dem 1. Oktober 1967 als Generalvikar fungierende Reinhard Lettmann, der sich nun unerwartet mit dem ,Fall Pottbäcker' konfrontiert sah. Als sich abzeichnete, dass „in absehbarer Zeit [...] ein Prozeß zu erwarten“ war, hielt er es „für notwendig“, ihn „aus der Seelsorge herauszuziehen und wenigstens vorläufig in einem geeigneten Haus unterzubringen, wo er auch priesterliche Hilfe erfährt". Da bereits zwei Jahre zuvor ein anderer Diözesanpriester „für längere Zeit“ bei den Kapuzinern in Werne aufgenommen und von diesen betreut worden war, wandte sich Lettmann nun "mit der gleichen Bitte" erneut an den dortigen Leiter, und nach dessen Zustimmung teilte er dem Kaplan wie auch - ,vertraulich“ - dem Dinslakener Pfarrer mit, dass es „nach Lage der Dinge“ für Pottbäcker „am besten“ sei, dort „bis auf weiteres“ zu wohnen“.28 Nachdem der Kapuziner-Provinzial in einem Telefonat mit dem Generalvikar jedoch das Kloster in Krefeld (Bistum Aachen) als das geeignetere ins Spiel gebracht hatte - die genauen Gründe sind nicht überliefert -, begab sich Pottbäcker schließlich Mitte November dorthin. ${ }^{29}$

Lettmanns Hoffnungen, Pottbäcker durch die Klosterunterbringung „ein wenig reifer zu machen“30, erfüllten sich offenbar zunächst nur zum Teil. Zumindest verband er Ende 1967 seine Mitteilung an den Kaplan über die offizielle Anklageerhebung mit der Bitte, „die Zeit im Kloster zur geistigen Besinnung und Weiterbildung zu nützen“, auf keinen Fall „wieder an den Ort [seiner] Tätigkeit in Dinslaken zurückzukehren“ und besonders „nicht zu vergessen", dass er allein schuld an seiner Situation sei. Dabei wies Lettmann ihn an, seine häufigen Reisen zu beenden und sich nicht über den „Aufenthalt bei den Patres“ zu beklagen, sondern deren „freundliche[s] Entgegenkommen [...]

27 Bösenseller Pfarrer an BGV vom 13.6.1967 (ebd., Bl. 110).

28 Lettmann an Definitor des Kapuziner-Klosters Werne vom 24.10.1967 (ebd., Bl. 108). Bereits 1963 war zudem der zuvor wegen sexuellen Missbrauchs an Messdienern im oldenburgischen Elisabethfehn zu einer Haftstrafe verurteilte Kaplan B. nach seiner Entlassung für drei Monate im Werner Kloster untergebracht gewesen, da er psychische Probleme hatte, vgl. Generalvikar Böggering an Offizial Grafenhorst vom 25.6.1963 (BAM, Offizialatsarchiv Vechta (OAV) A ol1). Dennoch dürfte das Kloster nicht als „Emeritenund Korrektionshaus“ gegolten haben, wie es Christine Hartig in ihrem Beitrag in diesem Buch für das Erzbistum Paderborn beschreibt.

29 Lettmann an den Definitor des Kapuziner-Klosters Werne vom 24.10.1967, an Pottbäcker und Dinslakener Pfarrer vom 6., 11. und 13.11.1967 sowie Kapuziner-Provinzial an Lettmann vom 16.11.1967 (Personalakte, Bl. 102-108).

30 Lettmann an Krefelder Guardian vom 15.7.1968 (ebd., Bl. 97). 
zu schätzen“. ${ }^{31}$ Bis zum Gerichtsprozess im Juni 1968 blieb Pottbäcker in Krefeld, also von der Bildfläche verschwunden.

Womöglich von der in Pottbäckers Bewährungsstrafe zum Ausdruck kommenden positiven Verhaltensprognose gestärkt, setzte Lettmann den Kaplan bereits einen Monat nach dem Urteilsspruch in Bockum-Hövel wieder in der Pfarrseelsorge ein. Doch scheint sich der Generalvikar durchaus des damit verbundenen Risikos bewusst gewesen zu sein. Zumindest informierte er den dortigen Pfarrer von der "Vorgeschichte“ seines neuen Mitarbeiters, ohne dass ersichtlich ist, wie konkret er den von Pottbäcker begangenen Missbrauch benannte. Da der Kaplan zudem eine Wohnung im Pfarrhaus bezog, sah Lettmann offenbar eine ausreichende Kontrolle gewährleistet, um erneuten Übergriffen vorzubeugen. ${ }^{32}$ Dass diese Maßnahmen nicht ausreichten, scheint jedoch lange Zeit nicht bis nach Münster gedrungen zu sein. Dafür spricht wenigstens die Verlängerung der seelsorglichen Vollmachten Pottbäckers für zweieinhalb Jahre, die Lettmann noch im Frühjahr 1971 aussprach. Ebenfalls bleibt unklar, warum er ihm - in wohlwollendem Ton - drei Monate später nach einem Gespräch mitteilte, bereits „in den nächsten Wochen mit einer Versetzung rechnen zu müssen“. ${ }^{33}$

Ob die Personalverantwortlichen des Bistums auch den Pfarrer der nachfolgenden Kaplanstelle in Rhede entsprechend instruierten, ist nicht überliefert. Jedenfalls dürften auch hier die bald einsetzenden Missbrauchstaten Pottbäckers kaum völlig unerkannt geblieben sein. So schildern Betroffene Situationen, in denen z.B. dem Küster/der Küsterin die von Pottbäcker in der Sakristei begangenen Verbrechen nicht verborgen geblieben sein können. Ebenso hätten während eines Ferienlagers die Betreuer, die sich meist aus dem Kreis der älteren Messdiener rekrutierten, aber auch die Kochfrauen mitbekommen, dass regelmäßig Jungen in Pottbäckers Zelt gingen. ${ }^{34}$ Dennoch konnte Pottbäcker Ende 1972 sein Pfarrexamen ablegen. ${ }^{35}$ Erst ein Jahr später führte schließlich die Beschwerde eines Vaters beim Ortspfarrer über Pottbäckers Übergriffe gegenüber seinem Sohn zur plötzlichen Abberufung des Kaplans.

\footnotetext{
$31 \quad$ Lettmann an Pottbäcker vom 29.12.1967 (ebd., Bl. 10o).

32 Lettmann an Bockumer Pfarrer und an Pottbäcker vom 25.6.1968 (ebd., Bl. 98f.).

33 Lettmann an Pottbäcker vom 4.8.1971 (ebd., Bl. 95); vgl. auch Beicht-Jurisdiktion Lettmanns vom 26.4.1971 und Lettmann an Bockumer Pfarrer vom 4.8.1971 (ebd., Bl. 96 und 94).

34 Interviews Franz Jeschke (Pseudonym) und Martin Schmitz vom 24.8. und 8.9.2020.

35 Pottbäcker an BGV vom 8.11.1972 (Personalakte, Bl. 93).
} 


\section{Vertrauen auf therapeutischen Erfolg (1974-1983)}

Spätestens jetzt dürfte der Bistumsleitung unter Bischof Heinrich Tenhumberg bewusst gewesen sein, dass die bislang verfolgte Strategie, den Problemen, die sich durch Pottbäckers pädosexuelle Präferenzstörungen ergaben, ausschließlich mit Versetzungen zu begegnen, nicht aufging und es daher weiterer Maßnahmen bedurfte. Erfolg versprach sie sich nun offenbar vor allem von einer therapeutischen Behandlung des Kaplans, wie es im Lauf der 1970er Jahre auch im Zusammenhang mit einigen ähnlich gelagerten ,Fällen` zu beobachten war. ${ }^{36}$ Bei Pottbäcker nahm der damalige Personalchef des Bistums, Wilhelm Stammkötter, daher Kontakt zum renommierten Leiter der Clemens-AugustKlinik in Neuenkirchen (im Oldenburger Münsterland) auf. Die nach Kardinal von Galen benannte Fachklinik für Psychotherapie, Neurologie und Innere Medizin besaß nicht nur eine enge Bindung zum Bistum Münster, sondern auch eine spezifische fachliche Expertise, sodass hier des Öfteren Diözesanpriester mit unterschiedlichen psychischen Problemen behandelt wurden. ${ }^{37}$ Nach einem Gespräch mit Pottbäcker - über Einzelheiten geben die Akten keine Auskunft - regte der Klinikleiter für ihn eine ambulante therapeutische Behandlung an, die der Kaplan schon bald bei einem im nördlichen Ruhrgebiet praktizierenden, entsprechend geschulten Arzt begann. ${ }^{38}$

Über diesen Arzt ließen sich bislang kaum konkrete Informationen finden. Ebenso wenig sind schriftliche Stellungnahmen oder Gutachten, wie sie etwa für Gerichtsverfahren erstellt wurden, ${ }^{39}$ überliefert, die die der Behandlung zugrunde liegende Diagnose sowie die Ziele und den Verlauf der Therapie dokumentieren könnten. Folgt man den Erinnerungen eines Pfarrers, der selbst zum heilkundlichen Therapeuten ausgebildet worden war, den Arzt kannte und sich mit ihm auf fachlicher Ebene austauschte, richtete der Arzt seine therapeutischen Bemühungen an den Grundsätzen Carl Gustav Jungs aus, der neben Sigmund Freud und Alfred Adler zu den Pionieren der Psychotherapie zählte und - etwa im Gegensatz zu Freud - gläubiger Christ war. Ferner habe Pottbäckers Therapeut die Meinung vertreten, dass Pädophilie

$3^{6} \mathrm{Zu}$ damit verbundenen Fragen - also etwa, wie im damaligen fachwissenschaftlichen Diskurs die „Heilungsperspektive“ bei pädosexuellen Präferenzstörungen eingeschätzt wurde, - sind die Recherchen noch nicht abgeschlossen.

Vgl. zur Klinik Clemens-August-Stiftung (Hrsg.), 50 Jahre Clemens-August-Stiftung. Dinklage 2003.

38 Stammkötter an Klinikleiter und an Therapeuten vom 12. und 26.10.1973 (Personalakte, Bl. 8gf.).

Vgl. etwa den Beitrag von Christine Hartig in diesem Buch. 
heilbar sei oder der Täter mindestens lernen könne, „sich zu kontrollieren“.40 Jedenfalls zählte dieser Arzt zu einem kleinen Kreis von Therapeuten, auf die die Bistumsleitung bei Bedarf zurückgriff, und zumindest für die Personalverantwortlichen des Bistums scheint nichts dagegen gesprochen zu haben, Pottbäcker neben einer Tätigkeit als Religionslehrer an einer Berufsschule in Recklinghausen als Subsidiar weiterhin eine Aufgabe in der Pfarrseelsorge zu geben. Auch wurde ihm erlaubt, einen eigenen Haushalt einzurichten. ${ }^{41}$

Ende 1979 bat Pottbäcker Personalchef Werner Thissen, der zwei Jahre zuvor an die Stelle Stammkötters getreten war, aus dem Schuldienst ausscheiden und in eine Pfarrstelle wechseln zu dürfen. Dabei erklärte er nach einer Aktennotiz des Personalchefs, dass seine langjährige therapeutische Behandlung diesen Schritt jetzt zulasse. Da Pottbäcker mittlerweile einen deutlich gefestigteren Eindruck auf den Personalchef machte und zudem der Therapeut in einem Telefonat, das der Personalchef mit Pottbäckers ausdrücklicher Zustimmung geführt hatte, sehr „befürwortet[e], daß P.Pfarrer wird“, ging Thissen schließlich auf Pottbäckers Wunsch ein. So wurde die Übernahme einer entsprechenden Pfarrstelle für die zweite Hälfte des nachfolgenden Jahres angestrebt. ${ }^{42}$ Doch dauerte es noch bis zum September 1981, ehe Pottbäcker in Recklinghausen als Pfarrer eingeführt wurde, wobei für die Verzögerung auch der Führerscheinentzug wegen wiederholter Autofahrten unter Alkoholeinfluss eine Rolle gespielt haben könnte. ${ }^{43}$

Im Frühjahr 1983 setzten die erneuten staatsanwaltschaftlichen Ermittlungen wegen sexueller Übergriffe Pottbäckers dieser von Therapeut und Personalverantwortlichen geteilten positiven Zukunftserwartung ein jähes Ende. Selbst Pottbäcker scheint seine Position in der Kirchengemeinde diesmal als unhaltbar betrachtet zu haben. Zumindest bat er Bischof Lettmann sehr schnell, ihn von seinem Amt zu entpflichten, was dieser auch umgehend umsetzte. ${ }^{44}$ Gleichzeitig lösten die Ereignisse bei den Bistumsverantwortlichen einen ähnlichen Mechanismus wie 15 Jahre zuvor aus, zumal die wegen Pottbäckers überraschender Entpflichtung zu erwartenden Nachfragen nicht zuletzt von Seiten der Presse - den Handlungsdruck deutlich erhöhten. Jedenfalls begab sich Pottbäcker bereits drei Tage später in das Priesterheim Johanneshöhe bei Neuwied am Rhein. In dieser 1969 von der Ordensgemeinschaft der Missionare vom heiligen Johannes dem Täufer gegründeten und

$40 \quad$ Interview Pfarrer B. vom 14.1.2020.

41 Vgl. BGV (Hauptabteilung Schule) an Pottbäcker vom 15.3.1974 und Stammkötter an Pottbäcker vom 15.3. und 28.11.1974 (Personalakte, Bl. 87f.).

42 Aktennotiz Thissens vom 14.12.1979 (ebd., Bl. 85).

43 Vgl. Urteil des Amtsgerichts Recklinghausen vom 9.7.1981 (ebd., Bl. $78 \mathrm{ff}$.).

44 Pottbäcker an Lettmann vom 26.4.1983 (ebd., Bl. 76). 
von den nordwestdeutschen Diözesen finanziell unterstützten Einrichtung erhielten Geistliche mit unterschiedlichen Schwierigkeiten geistliche und medizinische Hilfe. Gründe für die Unterbringung waren etwa psychische Erkrankungen oder Alkoholprobleme, aber auch die Verletzung des Zölibats bis hin zum sexuellen Missbrauch Minderjähriger. Je nach Bedarf wurden offenbar auch ein Facharzt für Neurologie und Psychiatrie konsultiert und bei anderen Fachleuten Gutachten eingeholt. ${ }^{45}$ Immerhin finanzierte die Diözese Münster Ende der 196oer Jahre auf der Johanneshöhe ein bis zwei „Priesterwohnungen“, und noch 1985 bedankte sich Lettmann beim Leiter des Priesterheims dafür, dass der Pater sich „in den letzten Jahren [...] mit großem Engagement einiger Priester unseres Bistums angenommen" hatte. Für die dabei entstandenen „mancherlei Aufwendungen“ überwies der Bischof zudem 5 ooo DM an die Ordensgemeinschaft. ${ }^{46}$

Allerdings dürfte es für die Bistumsverantwortlichen oftmals ebenso wichtig gewesen sein, dass „schwierige“ Geistliche auf der Johanneshöhe quasi von der Bildfläche verschwinden konnten. So basierten die Artikel, die Anfang Mai in den Regionalzeitungen über die Entpflichtung Pottbäckers berichteten, auch nur auf Vermutungen, die es jedoch „in sich“ hätten: der Pfarrer habe sich „sexuell an Minderjährigen vergangen“, betroffene Eltern hätten Strafanzeige erstattet. Selbst der Kirchenvorstand und der umgehend vom Generalvikariat in Münster zum Pfarrverwalter eingesetzte Geistliche könnten „den Anlaß für Pottbäckers Ausscheiden ebenfalls nur ahnen“, wobei "gravierende Gründe vorliegen“ müssten. „Was sich [...] wirklich zugetragen hat, wissen offenbar nur die Betroffenen. Pottbäckers Aufenthaltsort ist unbekannt, die übrigen Beteiligten schweigen. ${ }^{47}$ Allerdings habe Pottbäcker dem Pfarrverwalter mitgeteilt, dass er etwas getan habe, was er mit seinem „Amt nicht vereinbaren“ könne und eine Rückkehr in die Gemeinde ausschlösse. ${ }^{48}$ Wenig später berichteten die Zeitungen von der Anzeige der Eltern eines neunjährigen Jungen gegen Pottbäcker, wobei die Fakten nach Auskunft der Ermittlungsbehörde „sehr

45 Dafür sprechen zumindest einige Angaben in den wenigen, noch auf der Johanneshöhe verbliebenen Akten von Priestern, die im Bistum Münster tätig waren. Diese Akten gelangten 2011 nach dem Tod des seit ihrer Gründung die Einrichtung leitenden Paters ins BGV Münster (Akte Priesterheim Johanneshöhe, BAM, BGV Münster, HA 5oo, Reg. A o०53).

46 Lettmann an P. Sch. vom 23.7.1969 und 18.11.1985 (ebd.). Vgl. zur Johanneshöhe auch den Beitrag von Christine Hartig in diesem Buch.

47 Artikel „Pfarrer verläßt seine Gemeinde. Schwere Vorwürfe nicht geklärt“ und Meldung „Staatsanwaltschaft ermittelt gegen Priester" (Westdeutsche Allgemeine Zeitung vom 2. und 3.5.1983).

48 Artikel „Pfarrer Pottbäcker entpflichtet“ (Recklinghäuser Zeitung vom 3.5.1983). 
dünn“ seien und man daher mit „Verdächtigungen des Angeschuldigten sehr vorsichtig sein" solle. ${ }^{49}$

Zwei von Gemeindemitgliedern an Bischof Lettmann adressierte Briefe legen zudem nahe, dass es in der Pfarrei trotz der Beschuldigungen weiterhin Unterstützer Pottbäckers gab. In einem Antwortschreiben Thissens zeigte sich der Personalchef erfreut über die Stellungnahmen, wies auf die Notwendigkeit hin, Pottbäcker zu helfen, und bat daher zugleich um Verständnis, dass ihr ehemaliger Pfarrer im Augenblick keine direkten Kontakte wünsche. ${ }^{50}$ Darüber hinaus kam den Bistumsverantwortlichen ihr offenbar gutes Verhältnis zu den Justizbehörden zugute. Jedenfalls informierte der Pressereferent der Staatsanwaltschaft Bochum, die mit dem ,Fall Pottbäcker betraut war, Ende Mai 1983 Bischof Lettmann über eine für den nächsten Tag geplante Presseerklärung über die Beendigung der Ermittlungen. Der Beschuldigte sei "geständig und überführt", an mehreren neun- bis elfährigen Jungen „sexuelle Handlungen“ vorgenommen zu haben, wobei auf Grund des Geständnisses auf die Vernehmung der Kinder verzichtet werden konnte. Ferner habe die Staatsanwaltschaft "der Bildzeitung gegenüber auf die emotionale Relevanz in der Gemeinde" und die große Zurückhaltung der Eltern hingewiesen. Daher wäre es „nicht glücklich, wenn diese Sache gerade vor Fronleichnam noch einmal hochgespielt würde“. Schließlich ließ der Pressereferent den Bischof wissen, dass es eventuell anstatt „eines öffentlichen Verfahrens vor der Hauptstrafkammer" nur „zu einem schriftlichen Verfahren komme“. ${ }^{51}$ Mit dem Ende Juni 1983 gegen Pottbäcker ausgesprochenen Strafbefehl war zudem klar, dass ihm eine Haftstrafe, die er ebenso wie die Bistumsleitung vermutlich befürchtet hatte, erspart blieb.

\section{Raus aus der Pfarrseelsorge und unter stärkere Aufsicht $\left(1983^{-1987)}\right.$}

Jetzt konnten die Bistumsverantwortlichen konkrete Schritte für den weiteren Einsatz Pottbäckers einleiten. Zwar hatte sein Aufenthalt auf der Johanneshöhe in ihren Augen glücklicherweise dafür gesorgt, dass der "Schaden“ begrenzt

49 Meldung "Staatsanwaltschaft ermittelt gegen Priester“ (Recklinghäuser Zeitung vom 4.5.1983).

50 Thissen an Briefschreiber vom 19.5.1983 (Personalakte, Bl. 69 und 66).

51 Aktennotiz des Bischofssekretärs vom 31.5.1983 (Missbrauchsakte, BAM, BGV Münster, HA 5oo, Reg. A ooo9, Bl. 44); vgl. auch Artikel „Pfarrer legt Geständnis ab“ (Recklinghäuser Zeitung vom 1.6.1983). 
geblieben war. ${ }^{52}$ Dennoch führte sein ,Rückfall', der sich immerhin trotz mehrjähriger therapeutischer Behandlung ereignet hatte, zu einem erkennbaren Umdenken: Anstelle des Einsatzes im direkten Gemeindedienst wiesen sie ihm nun - zunächst für ein halbes Jahr angedacht - eine Tätigkeit im Bistumsarchiv zu, wobei er gleichzeitig wieder die Behandlung bei seinem langjährigen Therapeuten aufnehmen sollte. ${ }^{53}$ Bis Ende Juli blieb Pottbäcker noch auf der Johanneshöhe, um dann nach Münster umzuziehen. Hier musste er im Josefshaus eine Wohnung beziehen, das unter der Leitung von Vorsehungsschwestern stand. Es hatte zunächst als Haushaltungsschule mit Internat gedient, aber mittlerweile nutzte die Fachhochschule Münster die Lehräume und es wohnten ca. zehn Studentinnen im ehemaligen Internatstrakt, wobei der Konvent 20 bis 25 Schwestern zählte. ${ }^{54}$

Doch Pottbäcker fiel es offenbar schwer, sich mit der neuen Situation anzufreunden. Ihm war zwar klar - so eine Aktennotiz vermutlich Thissens vom Oktober 1984-, dass ihm die Übernahme einer ,selbständige[n] Aufgabe“ nicht möglich war, aber die Unterstützung eines Gemeindepfarrers könne er sich vorstellen, zumal er ja wieder eine Therapie begonnen habe und außerdem geistlichen Beistand bei einem anderen Priester suche. Wenn sich auch die aus Bischof, Generalvikar, Personalchef sowie dem Regens des Priesterseminars und den Weihbischöfen bestehende Personalkonferenz diesem Anliegen nicht verschloss und sich eine seelsorgliche Beschäftigung in einer Alteneinrichtung mit zusätzlichen "Sonntagsdiensten“ in einer Pfarrei vorstellen konnte, zerschlugen sich diese Pläne ebenso wie die nachfolgenden Bemühungen, Pottbäcker als Seelsorger in einer Klinik am Bodensee unterzubringen. ${ }^{55}$

Diese Anstrengungen erhielten 1986 einen deutlichen Dämpfer. Ob nach einem Hinweis der Vorsehungsschwestern oder von anderer Seite informiert, jedenfalls wies Thissen Pottbäcker in einem Gespräch eindringlich darauf hin, „daß er keine jungen Leute als Besucher in seiner Wohnung“ empfangen dürfe. Dabei „habe [Thissen] ihm deutlich vor Augen gestellt, was andernfalls daraus an Gerüchten entstehen könne und daß er im Falle eines Zuwiderhandelns

$52 \quad$ Thissen an P. Sch. vom 4.8.1983 (Personalakte, Bl. 49).

53 Leiter des BAM an Thissen vom 14.7.1983 und Aktenvermerk o. Verf. [vermutlich Thissen] vom 2.8.1983 (ebd., Bl. 5of.).

54 Vgl.zumJosefshausAngelikaWelzenberg,DieWestfälischeProvinzderOrdensgemeinschaft der Schwestern von der Göttlichen Vorsehung. Bd. 1: 1842 bis 1970. Münster 1992, 194f., 252 und 257f.; Jahresbericht des Schwesternkonvents des Josefshauses für 1986-88 (BAM, Mutterhausarchiv der Vorsehungsschwestern (MHA), Akte 622, Bl. 30-33) und „JosephEmilien-Stiftung: Abschied nach 108 Jahren“ (Münsterischer Anzeiger vom 2.6.199o).

55 Aktennotiz o. Verf. [vermutlich Thissen] vom 5.10.1984 mit handschriftlicher Zufügung, Thissen an Rektor Sp. vom 21.3.1985 und Sp. an Pottbäcker vom 11.7.1985 (Personalakte, Bl. 42, 39 und 35). 
dieser Abmachung nicht im Josefs-Haus bleiben könne“, was er auch der dortigen Oberin "mitgeteilt" habe. Pottbäcker gab sich einsichtig und versicherte dem Personalchef, „ab sofort keine jungen Leute mehr zu sich in seine Wohnung" zu lassen. ${ }^{56}$ Die Schwestern des Josefshauses scheinen jedenfalls ihre von Thissen zugewiesene Aufgabe, Pottbäcker im Auge zu behalten, sehr ernst genommen zu haben. Zumindest scheint sich Pottbäcker von den Schwestern überwacht gefühlt zu haben ${ }^{57}$ Aber auch die weitgehend berufsfremde Arbeit im Bistumsarchiv empfand er zunehmend als Belastung.

\section{Mitbrüderliches Agieren und ,Kapitulation` der Personalverantwortlichen $(1987-2007)$}

Im Herbst 1987 sah Pottbäcker den Zeitpunkt gekommen, den neuen Personalchef, der im Jahr zuvor dem zum Generalvikar ernannten Thissen nachgefolgt und sein ,Kurskollege' war, also seinem Weihejahrgang angehörte, um eine Tätigkeit etwa in der Krankenhaus-Seelsorge zu bitten, wobei der Personalchef sein „Kreuz“ berücksichtigen solle. Von diesem auf seine „Abmachung“ mit Thissen angesprochen, versicherte Pottbäcker, „daß eine akute Gefahr für ihn augenblicklich nicht bestehe“. So werde „das ganze Problem [...] ständig im Gespräch mit seinem Therapeuten [...] ,beackert“.. Inwieweit sich der Personalchef beim Arzt über Pottbäcker erkundigte, ist in der Personalakte nicht überliefert - schriftliche Stellungnahmen der Therapeuten ließen sich auch bei anderen Priestern nicht finden -, aber im April 1988 begann er als Seelsorger im Marien-Hospital in Rheinberg-Orsoy, das sich in Trägerschaft der münsterischen Clemensschwestern befand. Nach einem handschriftlichen Zusatz zur entsprechenden Aktennotiz waren die Oberin, der Dechant und der Bischöfliche Direktor der Ordensgemeinschaft „über die Situation“ informiert. 58

Keine zwei Jahre später scheint das Ergebnis einer Visitation durch Weihbischof Heinrich Janssen im Orsoyer Krankenhaus dem Personalchef vor Augen geführt zu haben, dass er sich nicht auf dieses, Sicherungsnetz' verlassen konnte. Jedenfalls erklärte er der Oberin nach einem „sehr offene[n] und ausführliche[n] Gespräch“ mit Pottbäcker, dass in dessen Wohnung „Besuche von

$56 \quad$ Aktennotiz Thissen vom 15.5.1986 (ebd., Bl. 34).

57 Vgl. Interview Simon Lederer (Pseudonym) vom 30.7.2020.

$5^{8}$ Aktennotiz des Personaldezernenten vom 1.10.1987 (Personalakte, Bl. 33); vgl. auch Diözesancaritasverband Münster (Hrsg.), Die caritativen Anstalten im Bistum Münster, Bd. 2. Münster o.J. [1955], 133ff. 
Kindern und Jugendlichen [...] nicht möglich“ seien. Sie solle dies „im Blick behalten“ und, „falls sie es im Laufe der Zeit für notwendig erachte, auf die eine oder andere Absprache Bezug zu nehmen“, „doch bitte unmittelbar und sofort mit Pfarrer Pottbäcker" und auch ihm sprechen. ${ }^{59}$ Schon bald erreichte die Bistumsleitung jedoch - so die Erinnerung eines mit den Vorgängen vertrauten Pfarrers - von einem Mann, der in Rheinberg wohnte und von Pottbäckers Vorstrafen wusste, die Aufforderung, ihn sofort aus Orsoy abzuberufen, da "Jungen-Fahrräder vor Pottbäckers Haustüre" stünden.60

Vielleicht dadurch ausgelöst, hatte Pottbäcker einen schweren, äußerst lebensbedrohlichen Herzinfarkt, der einen längeren Krankenhausaufenthalt erforderlich machte. ${ }^{61}$ Bereits während dieser Phase beschloss die Personalkonferenz Ende 199o, ihn aus Orsoy abzuziehen, was ihm - nach Aktenlage allerdings erst vier Monate später mitgeteilt wurde: Erneut sollte er nach Münster ziehen und sich im Bistumsarchiv betätigen. Pottbäcker zeigte sich über diese Entscheidung sehr betroffen, wies auf den eigentlich doch deutlichen Rückgang seiner Kinderbesuche hin und bemerkte, „wenn er zu nichts anderem gut sei, habe es sich wohl nicht gelohnt, wieder gesund zu werden“. Er halte es für das Beste, die Bistumsleitung ihm gegenüber „aus der Verantwortung [...] zu entlassen“ und "sich laisieren [zu] lasse[n]“, fügte aber direkt an, „daß das nur ein Gedanke sei, den er angesichts seiner Lebensberufung wohl gar nicht recht realisieren könne".62 Diese Argumentation, die auf das grundlegende Verständnis von Amt und Priestertum, aber auch des christlichen Verzeihens zielte und ein Appell an die Empathie des ,Kurskollegen' war, scheint möglicherweise den Personalchef so tief berührt $\mathrm{zu}$ haben, dass er ihm eine weitere Chance nicht verwehrte.

Auch wenn es nur schwer verständlich ist: Letztlich ,siegte die ausgeprägte priesterliche Mitbrüderlichkeit der Bistumsverantwortlichen über die Skepsis angesichts des sich seit 25Jahren regelmäßig wiederholenden Missbrauchs und distanzlosen Verhaltens gegenüber Kindern. Diesmal fand die Bistumsleitung für Pottbäcker im oldenburgischen Neuenkirchen in einer Suchtklinik eine neue Betätigungsmöglichkeit, wo der Wechsel des Seelsorgers anstand und sich das bislang erfolglose Muster der Information des direkten Umfeldes über Pottbäckers ,Haken' wiederholte. Selbst der psychotherapeutisch geschulte bisherige Amtsinhaber hoffte, dass „das Risiko wg. seiner Veranlagung“ durch

59 Personaldezernent an Oberin vom 28.8.1989 (Personalakte, Bl. 32).

6o Interview Pfarrer B. vom 14.1.2020.

61 Interview Simon Lederer (Pseudonym) vom 30.7.2020.

62 Aktennotiz des Personaldezernenten vom 27-3.1991 über ein Gespräch mit Pottbäcker am 26.3. in Münster (Personalakte, Bl. 3of.). 
die „nicht zu kleine Anzahl von Mitwissern“ gebremst werde und sich zudem die "gute Mitbrüderlichkeit" des damaligen Ortspfarrers - er gehörte ebenfalls zu Pottbäckers Weihekurs - positiv auswirke. ${ }^{63}$

Allerdings musste Pottbäckers Vorgänger bei einem unangekündigten Besuch feststellen, dass Pottbäcker "sofort wieder direkten Kontakt zu Jungen“ suchte und etwa Kinder der offenbar mit Zustimmung des Ortspfarrers von ihm geleiteten Messdienergruppe der Klinik „zum Spielen in seine Wohnung einlud“. Dabei habe er erlebt, wie Pottbäcker sich "auf die Ebene der Jungen begab, die ihn mit ,Heinzi' ansprachen“.64 Vielleicht durch solche Hinweise aufgeschreckt, versetzte ihn Lettmann Ende 1995 „ab sofort auch offiziell in den Ruhestand", sodass er nicht mehr als verantwortlicher Pfarrer in der Gemeinde-Seelsorge tätig werden konnte, und Anfang 1997 erinnerte der Personalchef den Neuenkirchener Ortspfarrer an die 1986 von Thissen mit „Pottbäcker getroffenen verbindlichen Absprachen“, obwohl er „in letzter Zeit nichts Negatives in dieser Hinsicht“ gehört und deshalb „eigentlich keinen Grund habe“, dem Pfarrer entsprechend zu schreiben. Pottbäcker wiederum versicherte, „kein Problem damit“ zu haben, die nötige Distanz zu Kindern zu wahren. ${ }^{65}$

Ein dreiviertel Jahr später zeigte ein ernüchternder Brief der Vorsitzenden des Klinik-Trägervereins an Lettmann, dass auch dieser Versuch, Pottbäcker wenigstens in der Krankenhaus-Seelsorge zu belassen, gescheitert war. Denn vor seiner Wohnung, die sich außerhalb des Klinikgeländes befand, wiesen Fahrräder sehr wohl auf häufigen Besuch von Jungen hin, und auch ohne einen Hinweis auf konkrete Übergriffe baten die Vorsitzenden den Bischof, Pottbäcker vollständig aus Neuenkirchen abzuziehen, um so nicht nur die Kinder und Jugendlichen, sondern auch Kirche wie Klinik und ebenfalls Pottbäcker „vor Schaden zu bewahren“. Gerade weil der Seelsorger „bei den Leuten, die in der Nachbarschaft der Klinik wohnen, sehr beliebt“ sei, könne „die Stimmung [...] sehr schnell und mit einer gewissen Radikalität umschlagen, sollte einmal ein Fall von Mißbrauch offenkundig werden“. Es gebe in Neuenkirchen „Bevölkerungskreise, die man als übersensibilisiert bezeichnen kann, die zu Überreaktionen neigen und die unsere Kirche und ihre Vertreter vor die Öffentlichkeit zerren. Die Lokalpresse greife, wie der Fall des schon lange verstorbenen Pfarrers Janzen lehrt, diese Dinge dankbar und schamlos auf." Die Vorsitzenden waren sich im Klaren, dass „es in Neuenkirchen auch andere

63 Pfarrer B. an Personaldezernent vom 5.1.1992 (ebd., Bl. 28f.).

64 Interview Pfarrer B. vom 14.1.2020.

65 Lettmann an Pottbäcker vom 30.11.1995, Personaldezernent an Ortspfarrer vom 23.1.1997 und Vermerk des Personaldezernenten vom 11.2.1997 (Personalakte, Bl. 22, 23 und 26). 
Sichtweisen der Problematik um Pfr. Pottbäcker“ gebe, meinten jedoch, „Verharmlosung und Verdrängung [seien] hier schlechte Ratgeber". Sie baten daher „um Verständnis für diese offene und vielleicht schonungslose Darstellung“, die sie gegenüber dem Bischof aber "schuldig zu sein“ glaubten. ${ }^{66}$

Welche Rolle die von den Vorsitzenden erwähnten, zwei Jahre zuvor sehr kontrovers geführten Diskussionen um die Benennung einer Schule nach dem langjährigen Neuenkirchener Pfarrer Bernhard Janzen bei der Entscheidungsfindung des Bischofs gespielt haben - Betroffene hatten in diesem Zusammenhang erstmals den in den 195oer/6oer Jahren selbst erlebten massiven sexuellen Missbrauch durch den hoch angesehenen Pfarrer öffentlich gemacht $t^{67}$-, ist nicht überliefert. Aber bereits wenige Tage später erfolgte die fristlose Kündigung Pottbäckers durch die Klinik, und auch Bischof Lettmann ordnete trotz Widerständen in der Gemeinde an, dass Pottbäcker den Ort zu verlassen habe. So ließ er sich auch nicht mehr durch das Argument des Ortspfarrers umstimmen, das von Pottbäcker ausgehende Risiko sei grundsätzlich „an einem anderen Ort genauso“ groß. ${ }^{68}$ Auch in Teilen der Neuenkirchener Gemeinde stieß der Schritt auf Unverständnis, zumal sie über die wahren Gründe weitgehend in Unkenntnis gelassen wurde, wie es auch in einem Artikel der Kirchenzeitung zum Ausdruck kam. Dort hieß es nach einer äußerst positiven Schilderung über Pottbäckers seelsorgliche Tätigkeit: „Das ist seine Art: schlicht, einfach, humorvoll. Wohl deshalb wird er von Kindern, Jugendlichen und Erwachsenen hier so geschätzt." ${ }^{\text {"69 }}$

Das weitere Vorgehen der Bistumsverantwortlichen - nicht zuletzt Bischof Lettmanns - im, Fall Pottbäcker' zeugt von großer Ratlosigkeit und mangelnder Konsequenz. So bezog Pottbäcker im Frühjahr 1998 in einer Kirchengemeinde der Bischofsstadt eine Wohnung. Als zunächst einzige dienstliche Verpflichtung stand an jedem Samstagmorgen in einem außerhalb der Stadt liegenden Klarissen-Kloster die Gottesdienstfeier auf dem Plan. Ausdrücklich wies ihn der Personaldezernent zwar erneut „auf unsere verbindliche Absprache hin, daß er auch weiterhin Kinder und Jugendliche nicht in seiner Wohnung empfängt.“70 Gleichwohl sollte er schon bald zusätzlich „sporadisch

66 Vorsitzende des Münsterländischen Volksheilstättenvereins e.V. zu Vechte an Lettmann vom 20.11.1997 (ebd., Bl. 2of.).

67 Vgl. etwa Artikel „Die Opfer schwiegen mehr als zo Jahre“ (Oldenburgische Volkszeitung vom 8.7.1995).

68 Ortspfarrer an Lettmann vom 27.11./9.12.1997 und Sekretariat Lettmanns an Personaldezernent vom 15.12.1997 (Personalakte, Bl. 15ff. bzw. ohne Paginierung).

69 Artikel „Musik in der Predigt. Pfarrer Heinz Pottbäcker verläßt Neuenkirchen“ (Kirche und Leben, Regionalteil Oldenburg vom 18.1.1998).

70 Aktennotiz des Personaldezernenten vom 29.4.1998 (Personalakte, Bl. 12). 
anfallende Aushilfstätigkeiten in verschiedenen Gemeinden des Bistums“ übernehmen. ${ }^{71}$ Für die nachfolgenden Jahre bis zu Pottbäckers Tod im Jahr 2007 enthält die ansonsten vergleichsweise dicht geführte Personalakte keine weiteren Angaben mehr, und auch sonst konnte bislang nicht geklärt werden, ob und in welchem Umfang etwa die Pfarrer, Kirchenvorstände und Pfarrgemeinderäte in seiner neuen Wohngemeinde über seine pädosexuellen Präferenzstörungen und Vorstrafen informiert wurden.

Weiterhin wurde Pottbäcker also das Zelebrieren von Messen nicht verboten. Er machte nach den Erinnerungen seines Vorgängers in Neuenkirchen „überall im Bistum fleißig Vertretung “. ${ }^{72}$ Die Gottesdienste und den Umgang mit den Klarissen scheint er zwar nicht als erfüllend und unkompliziert empfunden zu haben, doch letztlich sei er "gut mit ihnen ausgekommen“, wie er einer ihm nahestehenden Person berichtet hat. ${ }^{73}$ Wirkliche Kontrollinstanzen installierten die Bistumsverantwortlichen nach Aktenlage jedenfalls nicht mehr. Seine letzte Ruhestätte fand Pottbäcker auf dem Zentralfriedhof in Münster. Auf dem wie üblich vom Bistum veröffentlichten und von Lettmann unterschriebenen Totenzettel finden sich keine Hinweise auf wie auch immer geartete Probleme, die Pottbäckers Priesterleben begleitet haben. ${ }^{74}$

\section{Fazit}

Allein schon wegen der immensen Zahl der pädokriminellen Verbrechen Pottbäckers, die für viele der Betroffenen z.T. schwere, bis in die Gegenwart reichende traumatisierende Folgen haben, ist dem ,Fall' eine besondere Bedeutung beizumessen. Neben den 21 Betroffenen, für die es in den Akten und Zeitungsberichten konkrete Hinweise gibt - sie betreffen den Zeitraum von 1967 bis $1983^{-}$, ist von einer sehr hohen Dunkelziffer auszugehen, da Pottbäcker im Rahmen seiner engagierten Jugendarbeit vielfältige Möglichkeiten hatte, seine Taten anzubahnen und durchzuführen. Dabei war seine gerade von den jüngeren Mitgliedern der Gemeinden äußerst positiv empfundene Ausstrahlung ein wesentlicher Bedingungsfaktor. Als im Gottesdienst Gitarre spielender Geistlicher hob er sich in den 1970er Jahren deutlich von der älteren Priestergeneration ab, sodass er schnell und problemlos die Distanz

71 Personaldezernent an Pottbäcker vom 22.5.1998 (ebd., Bl. 11).

72 Interview Pfarrer B. vom 14.1.2020.

73 Interview Simon Lederer (Pseudonym) vom 30.7.2020.

74 Totenzettel Pottbäckers vom 13.2.2007 (Personalakte, Bl. 3-7). 
zu Kindern und Jugendlichen abbauen konnte. Dies schmälerte jedoch nicht seine nach wie vor qua Amt bestehende priesterliche Autorität, die offenbar nicht selten zu einer Kultur des Wegschauens und ,Nichtglaubenwollens bei Eltern und Funktionsträgern der Gemeinden führte. Der Umfang dieses ,impliziten Wissens‘ sollte nicht unterschätzt werden.

Zum erschreckenden Ausmaß der Missbrauchstaten Pottbäckers trug wesentlich die Versetzungspraxis der Bistumsleitung bei, die Pottbäcker trotz ihres Wissens um seine pädosexuellen Präferenzstörungen und Missbrauchstaten mehr als 15 Jahre in der Gemeindeseelsorge beließ. Erst der Strafbefehl von 1983 führte hier zu einem Umdenken. Aber auch die bereits 1974 eingeleitete therapeutische Behandlung wie das 1986 erstmals installierte Kontrollsystem, bei dem nicht nur das jeweilige direkte Umfeld Pottbäckers von seiner "Vorgeschichte“ informiert, sondern ausdrücklich auch um Mitteilung bei Vorfällen angehalten wurde, konnte ihn niemals wirklich davon abhalten, immer wieder aktiv die Nähe von Kindern zu suchen. Da in keinem der in der Personalakte zu findenden Schreiben und Vermerke der Personalverantwortlichen die Missbrauchshandlungen Pottbäckers konkret benannt werden - Detailwissen war ihnen aus den Anklageschriften und dem Gerichtsurteil sehr wohl bekannt -, ist allerdings zu vermuten, dass die ,Kontrolleure` auf Grund dieser auch im Zusammenhang mit anderen Tätern festzustellenden großen ,Sprachlosigkeit‘ nur rudimentäre Vorstellungen der tatsächlich von Pottbäcker ausgehenden Gefahren besaßen. Letztlich beließ die Bistumsleitung Pottbäcker trotz zahlreicher Rückfälle im seelsorglichen Dienst. Eine Suspendierung scheint jedenfalls niemals ernsthaft in Erwägung gezogen worden zu sein.

Bei allen Maßnahmen, die letztlich nie den kirchlich bestimmten Raum verließen, hatte zunächst der Schutz der Institution oberste Priorität. Dies kam etwa zum Ausdruck, als Pottbäcker während der staatsanwaltschaftlichen Ermittlungen in speziellen Einrichtungen untergebracht wurde. Diese boten zwar eine geistliche und zum Teil auch medizinische Begleitung, sollten aber doch zuvorderst Pottbäcker aus der Schusslinie nehmen. Selbst bei den 1983 auch in der Öffentlichkeit diskutierten Vorfällen galt bewusste Intransparenz als das entscheidende Mittel, um „Schaden“ abzuwenden.

Allerdings sollte ebenfalls in Betracht gezogen werden, ob nicht auch von Seiten der Justiz durch das Aussetzen der Gefängnisstrafe auf Bewährung im Gerichtsurteil von 1968 und der damit in den Augen der Richter verbundenen positiven Sozialprognose oder durch den Strafbefehl von 1983 anstatt einer Gerichtsverhandlung eine konsequentere Vorgehensweise verhindert wurde. Zudem verfolgte die Staatsanwaltschaft 1983 im Zuge ihrer Ermittlungen eine Informationsstrategie, die der Bistumsleitung bei ihrem Bemühen 
entgegenkam, einer breiteren Auseinandersetzung mit den Geschehnissen aus dem Weg zu gehen.

Die 1973 von den Personalverantwortlichen unter Bischof Tenhumberg mit großen Hoffnungen eingeleitete psychotherapeutische Behandlung fand wie üblich bei einem kirchennahen Arzt statt, der offenbar grundsätzlich davon ausging, dass ein pädophiler Täter dazu geführt werden könne, keine Übergriffe mehr zu verüben. Immerhin befürwortete er nach fünf Jahren ausdrücklich Pottbäckers Übernahme einer Pfarrstelle, und auch für den weiteren Verlauf der nach einem erneuten Rückfall Pottbäckers durchgeführten Therapie gibt es keinerlei Hinweise, dass er auf dessen konsequente Herausnahme aus den seelsorglichen Bezügen gedrungen hat. Letztlich wurde auf diese Weise, wie auch bei weiteren Fällen übergriffiger Priester, die Verantwortung verlagert und so die Bistumsleitung entlastet.

Im ,Fall Pottbäcker' lag ein Großteil dieser Verantwortung bei Reinhard Lettmann, der zwischen 1967 und 2008 als Generalvikar, Weihbischof und Bischof mit dem Sachverhalt konfrontiert war. Aber auch die jeweiligen Personaldezernenten und andere beteiligte Geistliche zeigten im Umgang mit Pottbäcker immer wieder eine zu weit gehende Fürsorge, indem sie ihn zuerst als ihren priesterlichen, Mitbruder sahen. Dabei erwies sich gerade die Solidarität unter ,Kurskollegen` als besonders fest, sodass Pottbäcker innerhalb dieser ,männerbündischen' Strukturen durchaus Empathie einfordern und erwarten konnte. Wie zumindest im Zusammenhang mit dem von ihm selbst angesprochenen Gedanken einer Laisierung zu vermuten, spielte hier auch eine Rolle, vor dem Hintergrund der spezifischen Bedeutung des Weiheamtes - also des Priestertums als ,Lebensaufgabe - seine Priesterberufung zu retten. Auch scheint es für die Bistumsleitung zu keinem Zeitpunkt ein Thema gewesen zu sein, kirchenrechtliche Ermittlungen anzustoßen. Im Gegenteil sprechen die letzten Priesterjahre Pottbäckers für Lettmanns Unvermögen, wirklich konsequente Schritte einzuleiten. Letztlich kapitulierte er vor Pottbäckers pädosexuellen Präferenzstörungen.

Wenn 1997 im Beschwerdebrief des Neuenkirchener Klinikträgers erstmals davon die Rede ist, dass es auch einen „Schaden“ an Kindern und Jugendlichen zu vermeiden gelte, weist dies deutlich auf den lange Zeit völlig fehlenden Blick auf die Jungen und Mädchen hin, die von Pottbäckers Missbrauchstaten betroffen waren. Diese mangelnde Empathie ist jedoch nicht nur für die Bistumsleitung und die involvierten Geistlichen, sondern auch für die zuständigen Justizbehörden und Gemeinden Pottbäckers zu konstatieren, wo das durchaus vorhandene Wissen zumindest vom distanzlosen Verhalten des Kaplans nicht dazu geführt hat, sich schützend vor die Kinder zu stellen. Auch deshalb kann der ,Fall Pottbäcker' als Menetekel dienen. 